\title{
Static Contract Checking for Haskell
}

\author{
Dana N. Xu \\ University of Cambridge \\ $\mathrm{n} \times 200 @$ cam.ac.uk
}

\author{
Simon Peyton Jones \\ Microsoft Research \\ simonpj@microsoft.com
}

\author{
Koen Claessen \\ Chalmers University of Technology \\ koen@chalmers.se
}

\begin{abstract}
Program errors are hard to detect and are costly both to programmers who spend significant efforts in debugging, and for systems that are guarded by runtime checks. Static verification techniques have been applied to imperative and object-oriented languages, like Java and C\#, but few have been applied to a higher-order lazy functional language, like Haskell. In this paper, we describe a sound and automatic static verification framework for Haskell, that is based on contracts and symbolic execution. Our approach is modular and gives precise blame assignments at compile-time in the presence of higher-order functions and laziness.
\end{abstract}

Categories and Subject Descriptors D.3 [Software]: Programming Languages

General Terms verification, functional language

Keywords contract satisfaction, static contract checking

\section{Introduction}

Program errors are common in software systems, including those that are constructed from functional languages, and much research attention has been paid to the early and accurate detection of such errors. Formulating and checking (dynamically or statically) logical assertions [22, 13, 4], especially in the form of contracts [24, 2], is one popular approach to error discovery. Assertions state logical properties of an execution state at arbitrary points in the program; contracts specify agreements concerning the values that flow across a boundary between distinct parts of a program (modules, procedures, functions, classes). In functional languages, the presence of higher-order values and lazily-constructed values complicate assertion and contract checking, but considerable progress has been made, especially for dynamically-checked contracts $[11,10,18]$. In addition, recent proposals have introduced static pre/post-condition checking and hybrid (mixed static/dynamic) contract checking for functional languages $[38,12,21,20,16]$.

In this paper, we present a sound and automatic method for static contract checking for a higher-order lazy functional language, Haskell, by combining the ideas of higher-order contract semantics [11,3] and static verification through symbolic execution [38]. Consider:

$$
\begin{aligned}
& f:: \text { [Int] } \rightarrow \text { Int } \\
& f x s=\text { head } x s \text { ' } m a x \text { ' } 0
\end{aligned}
$$

where head is defined in the module Prelude as follows:

$$
\begin{aligned}
\text { head ::[a] } & \rightarrow \mathrm{a} \\
\text { head }(\mathrm{x}: \mathrm{xs}) & =\mathrm{x} \\
\text { head }[] & =\text { error "empty list" }
\end{aligned}
$$

If we have a call ( $f[]$ ) in our program, its execution will result in the following error message:

\section{Exception: Prelude.head: empty list}

This gives no information on which part of the program is wrong except that head has been wrongly called with an empty list. Presumably, the programmer's intention is that head should not be called with an empty list. To express this intention, programmers can give a contract to the function head. Contracts are implemented as pragmas:

$\{-\#$ CONTRACT head $::\{s \mid$ not (null s) $\}->\{z \mid$ True $\} \#-\}$ where not and null are just ordinary Haskell functions:

$$
\begin{array}{lll}
\text { null :: }[\text { a }] \rightarrow \text { Bool } & \text { not : : Bool } \rightarrow \text { Bool } \\
\text { null }[]=\text { True } & \text { not True }=\text { False } \\
\text { null xs }=\text { False } & \text { not False }=\text { True }
\end{array}
$$

This contract places the onus on callers of head to ensure that the argument to head satisfies the expected precondition. With this contract, our compiler would generate the following warning (by giving a counter-example) when checking the definition of $f$ :

$$
\text { Error: } f \text { [] calls head }
$$$$
\text { which fails head's precondition! }
$$

This paper makes the following specific contributions:

- We give a crisp, declarative specification for what it means for a term to satisfy a contract $(\S 4)$. This is unusual, with the notable exception of Blume \& McAllester [3].

- Unlike Blume \& McAllester and most other related work on higher-order contracts, we focus on static verification, and target a lazy language.

- Our contracts themselves contain unrestricted Haskell terms, so we tackle head-on the question of what happens if the contract itself crashes $(\S 6)$ or diverges $(\S 7)$.

- Despite this generality, we are able to give a very strong theorem expressing the soundness and completeness of contract wrappers as compared to contract satisfaction $(\S 5)$.

- We develop a concise notation ( $\triangleright$ and $\triangleleft$ ) for describing contract checking, that enjoys many useful properties $(\S 5.3)$. Thus equipped, we give a new proof of the soundness and completeness of contract wrappers. This proof is quite simple, but setting up the design to make it simple was much trickier than we expected.

- Our framework neatly accommodates some subtle points that others have encountered, including: ensuring that all contracts are inhabited ( $\$ 4.3)$, and the Any contract $(\S 4.4)$.
Permission to make digital or hard copies of all or part of this work for personal or
classroom use is granted without fee provided that copies are not made or distributed for profit or commercial advantage and that copies bear this notice and the full citation on the first page. To copy otherwise, to republish, to post on servers or to redistribute to lists, requires prior specific permission and/or a fee.

POPL'09, January 18-24, 2009, Savannah, Georgia, USA.

Copyright (C) 2009 ACM 978-1-60558-379-2/09/01...\$5.00. 
- We describe how to augment the contract decision procedure so that it maintains extra information that helps the programmer to localise the error $(\S 8)$.

\section{Overview}

The type of a function constitutes a partial specification to the function. For example, sqrt : : Int $\rightarrow$ Int says that sqrt is a function that takes an integer and returns an integer. A contract of a function gives more detailed specification. For example: $\{-\#$ CONTRACT sqrt $::\{x \mid x>=0\} \rightarrow\{z \mid z<=x\} \#-\}$ says that the function sqrt takes a positive value and returns a value that is smaller than the input. A contract can therefore be viewed as a refinement to a type; it is therefore also known as refinement type in $[15,7,12]$.

This paper describes a system that allows a programmer to write a contract on some (but, like type signatures, not necessarily all) definitions, and then statically checks whether the definition satisfies the contract. This check is undecidable, and so may give the result "definitely satisfies", "definitely does not satisfy", or "don't know". In the latter two cases we emit information that helps to localise the (possible) bug. We begin, however, by giving the flavour of contracts themselves with various examples, concentrating on aspects where our approach differs from other contract systems. $\S 4$ makes contracts precise.

\subsection{Base and Dependent Function Contracts}

We adopt the basic contract notation from [11, 12]. For example, here is a contract that declares that the length of the result rs is the same as the length of the argument $\mathrm{xs}$ (where $\mathrm{Ok}$ is short for $\{\mathrm{x} \mid$ True $\}$ ):

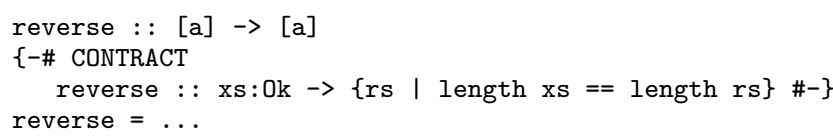

The expression (length $\mathrm{xs}==$ length $\mathrm{rs}$ ) is an arbitrary boolean-valued Haskell expression. Programmers do not need to learn a new language of predicates; they just use Haskell.

Notice too that this is a dependent function contract, because the argument $x s$ is used in the result contract.

The contract notation is more expressive than the requires, ensures notation used in our earlier work [38], because it scales properly to higher-order functions. Consider an example adapted from [3]:

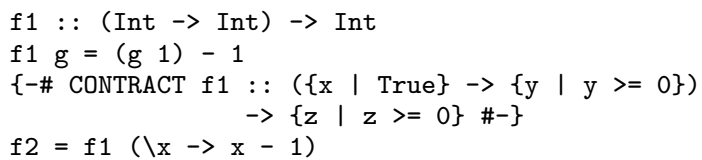

The contract of $f 1$ says that if $f 1$ takes a function that returns a natural number when given any integer, the function $f 1$ itself returns a natural number.

The Findler-Felleisen algorithm in [11] (a dynamic contract checking algorithm) can detect a violation of the contract of $f 1$. However, it cannot tell that the argument of $f 1$ in the definition of $f 2$ fails $f 1$ 's precondition (due to the lack of witness at run-time). On the other hand, the Sage system in [21] (a hybrid contract checking system) can detect the failure in $\mathrm{f} 2$ statically, and can report contract violation of $f 1$ at run-time. Our system reports both failures at compile-time with the following messages:

Error: f1's postcondition fails when $(\mathrm{g} 1)>=0$ holds (g 1$)-1>=0$ may not hold

Error: $f 2$ calls $f 1$

which fails $f 1$ 's precondition

\subsection{Laziness}

Laziness can cause false alarms. For example:

$$
\begin{aligned}
& \text { fst }(a, b)=a \\
& f 3=\text { fst }(5, \text { error " } f ")
\end{aligned}
$$

Syntactically, the call fst (5, error " $f$ ") appears unsafe because the existence of a call to error, but in a lazy language like Haskell the call is perfectly safe. The only static verification tool that caters for laziness is the ESC/Haskell system [38], which can reduce false alarms due to laziness by inlining. In the above case, the function $\mathrm{fst}$ is inlined, so the call to $\mathrm{fst}$ in $\mathrm{f} 3$ becomes 5 which is syntactically safe. However, if the size of the lazy function is big, or the function is recursive, the inlining strategy breaks down. In this paper we therefore introduce a special contract Any, which every expression satisfies. Now we can give f st a contract

$$
\{-\# \text { CONTRACT fst : : (Ok, Any) } \rightarrow \text { Ok \#-\} }
$$

which says that fst does not care what the second component of the argument is, as long as the first component is crash-free, the result is crash-free. Here, with the contract Any, without inlining any function, our system can tell that $f 3$ is safe.

This means that we give a crashing expression (such as error "msg") the contract Any, while in [3] an expression that unconditionally crashes satisfies no contract. This is one of the key differences in designing the contract semantics.

\subsection{Data Constructors in Contracts}

In the previous section we gave fst's argument the contract (Ok, Any); that is, the argument should be a pair whose first component satisfies $0 \mathrm{k}$, and whose second satisfies Any. We generalise this form for any user-defined data constructor. For example:

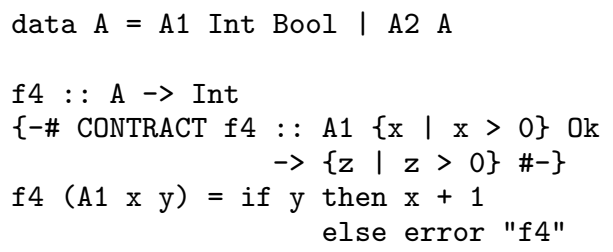

Note that, the data constructor $\mathrm{A} 1$ is used in the above contract while the data type $\mathrm{A}$ is used in the type specification. Besides checking the sub-components of $\mathrm{A} 1$, a call ( $f 4$ (A2 z ) ) is rejected because the contract of $f 4$ says that $f 4$ can only be applied to data constructed by $\mathrm{A} 1$.

\subsection{Partial Functions in Contracts}

A partial function is one that may crash or diverge. For example, the function head crashes when given an argument []. Since we allow arbitrary Haskell code in contracts, what are we to say about contracts that crash or diverge? One possibility is to simply exclude all such contracts - but excluding divergence requires a termination checker, and excluding functions like head is extremely restrictive. For example:

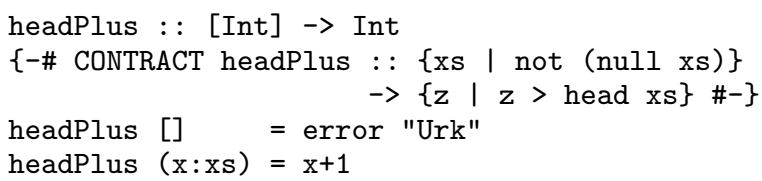

Here the postcondition uses head (which may crash), but that seems entirely reasonable in view of the precondition that $\mathrm{xs}$ is 
non-empty. Nevertheless, such a contract is rejected by [3], because of the call to head.

Our approach is to permit divergence in contracts (which avoids the requirement for a termination checker), but to require them to be "crash-free". Our definition of crash-free-ness for contracts takes account of dependency, and hence is much more liberal than requiring each Haskell term in the contract to be independently crash-free (which excludes head). This liberality is, we believe, key to making contracts usable in practice. We discuss crash-freeness of contracts in $\S 6.1$ and divergence in $\S 7.1$.

\subsection{The Plan for Verification}

It is all very well for programmers to claim that a function satisfies a contract, but how can we verify the claim statically (i.e. at compile time)? Our overall plan, which is similar to that of Blume \& McAllester [3], is as follows.

- Our overall goal is to prove that the program does not crash, so we must first say what programs are, and what it means to "crash" (§3.3).

- Next, we give a semantic specification for what it means for an expression $e$ to "satisfy a contract" $t$, written $e \in t(\S 4)$.

- From a definition $f=e$ we form a term $e \triangleright t$ pronounced " $e$ ensures $t$ ". This term behaves just like $e$ except that (a) if $e$ disobeys $t$ then the term crashes; (b) if the context uses $e$ in a way not permitted by $t$ then the term loops. The term $e \triangleright t$ is essentially the wrapper mechanism first described by Findler \& Felleisen [11], with some important refinements (§5).

- With these pieces in place, we can write down our main theorem for crash-free contracts $t(\S 5)$, namely that

$$
e \in t \quad \Longleftrightarrow \quad(e \triangleright t) \text { is crash-free }
$$

We must ensure that everything works properly, even if $e$ diverges, or laziness is involved, or the contract contains divergent or crashing terms.

- Using this theorem, we may check whether $f \in t$ holds as follows: we attempt to prove that $(e \triangleright t)$ is crash-free - that is, does not crash under all contexts. We conduct this proof in a particularly straightforward way: we perform symbolic evaluation of $(e \triangleright t)$. If we can simplify the term to a new term $e^{\prime}$, where $e^{\prime}$ is syntactically safe - that is, contains no crashes everywhere in the expression - then we are done. This test is sufficient, but not necessary; of course, the general problem is undecidable.

\section{The Language}

The language presented in this paper, named language $\mathcal{H}$, is simply-typed lambda calculus with case-expression, constructors and integers. Language $\mathcal{H}$ is simpler than the language we use in our implementation, which is the GHC Core Language [33], which is similar to System F and includes parametric polymorphism.

\subsection{Syntax}

The syntax of our language $\mathcal{H}$ is shown in Figure 1. A program is a module that contains a set of data type declarations, contract specifications and function definitions. Expressions include variables, term abstractions and applications, constructors and case expressions. We treat let-expressions as syntactic sugar:

$$
\text { let } x=e_{1} \text { in } e_{2} \quad \equiv_{s} \quad\left(\lambda x . e_{2}\right) e_{1}
$$

We omit local letrec, in favour of recursive (or mutually recursive) top-level functions. The language is typed, and to save clutter in this paper we silently assume that all expressions, contexts and

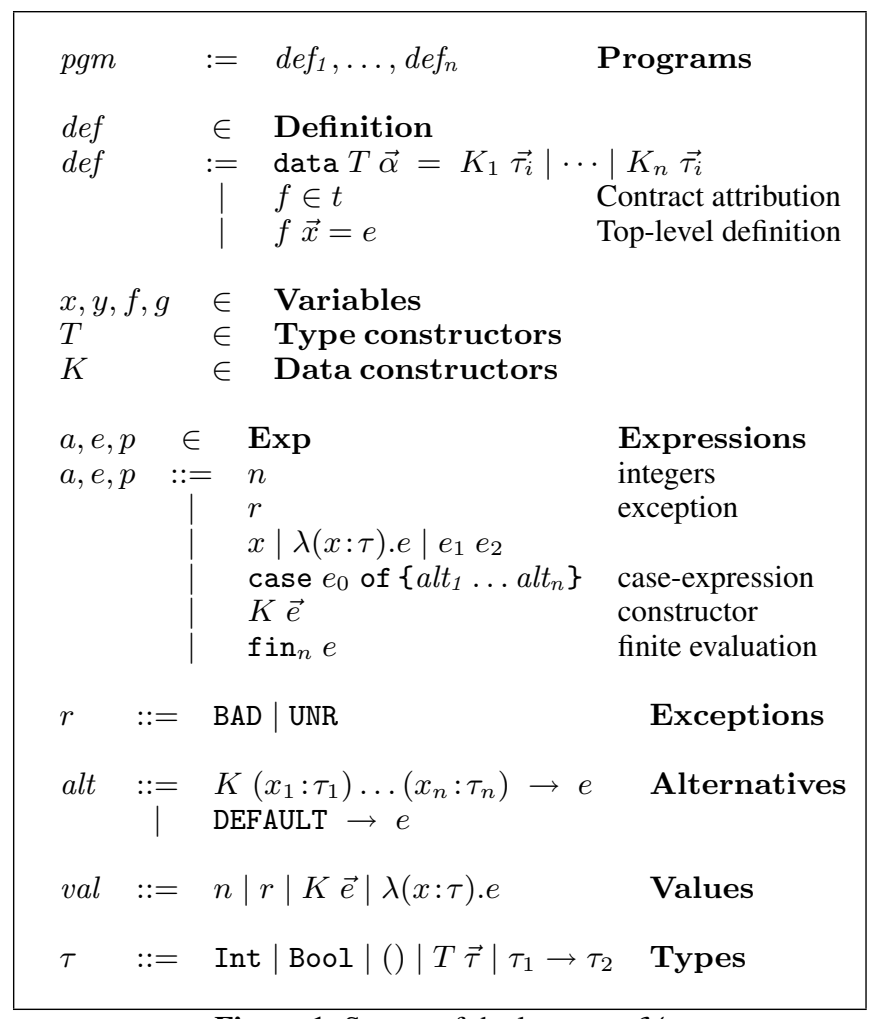

Figure 1: Syntax of the language $\mathcal{H}$

contracts are well-typed. Type checking for contracts can be found in [39].

There are two exception values adopted from [38]:

$\mathrm{BAD}$ is an expression that crashes. A program crashes if and only if it evaluates to BAD. For example, a user-defined function error can be explicitly defined as:

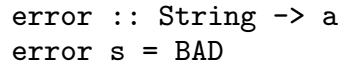

A preprocessor ensures that source programs with missing cases of pattern matching are explicitly replaced by the corresponding equations with BAD constructs. For example, after preprocessing, function head's definition becomes:

$$
\begin{array}{ll}
\text { head }(x: x s) & =x \\
\text { head }[] & =B A D
\end{array}
$$

UNR (short for "unreachable") is an expression that gets stuck. We use it to make the program halt when its context has misbehaved - it is unreachable when the context is well-behaved. Hence UNR is not considered a "crash". A program that loops forever also does not crash, and does not deliver a result, so you can think of UNR as a term that simply goes into an infinite loop.

\subsection{Operational Semantics}

The semantics of our language is given by the confluent, nondeterministic rewrite rules in Figure 2. We use a small-step reductionrule semantics, rather than (say) a deterministic more machineoriented semantics, because the more concrete the semantics becomes, the more involved the proofs become too.

Most of these rules are entirely conventional. The rule [E-top] deals with a top-level function call $f$. We fetch its definition from the environment $\Delta$, which maps a variable to its type, contract and definition. To save clutter, we usually leave this environment 


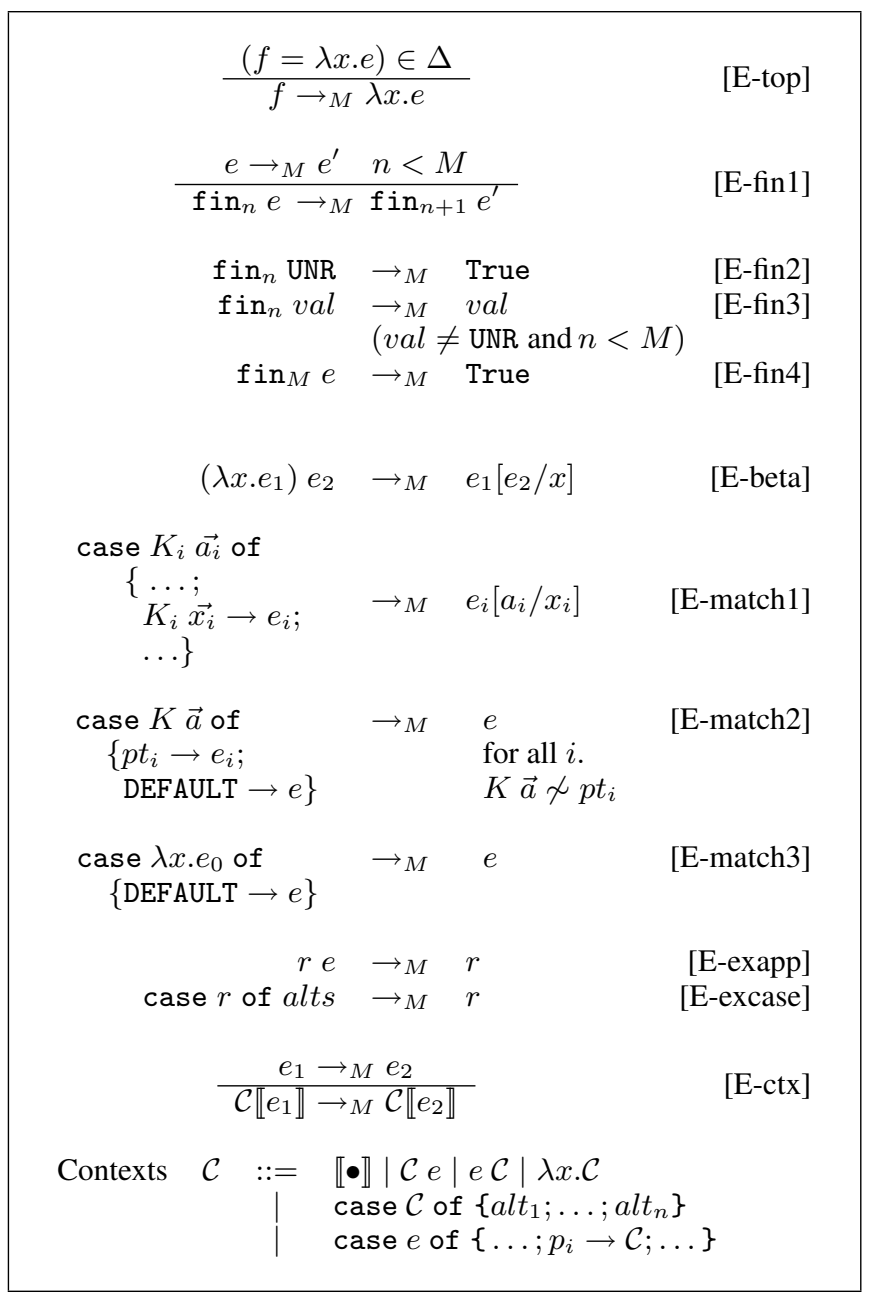

Figure 2: Semantics of the language $\mathcal{H}$

implicit, rather than writing (say) $\Delta \vdash e_{1} \rightarrow_{M} e_{2}$. Rules [E-exapp] and [E-excase] deal with exception values in the usual way. Rule [E-ctx] allows a reduction step to take place anywhere. The relation $e_{1} \rightarrow_{M} e_{2}$ performs a single step reduction and the relation $\rightarrow_{M}^{*}$ is the reflexive-transitive closure of $\rightarrow_{M}$.

The unconventional features are the " $M$ " subscript on the reduction arrow, the form $\mathrm{fin}_{n} e$, and the reduction rules [E-fin1-4]. These aspects all concern divergence, and are discussed in detail in $\S 7.1$, where we define $\rightarrow^{*}$ in terms of $\rightarrow_{M}^{*}$. For the present, simply ignore the subscripts and $\mathrm{fin}$.

Now we can give the usual definition of contextual equivalence:

DEFINITION 1 (Semantically Equivalent). Two expressions $e_{1}$ and $e_{2}$ are semantically equivalent, namely $e_{1} \equiv_{s} e_{2}$, iff

$$
\forall \mathcal{C}, r . \quad \mathcal{C} \llbracket e_{1} \rrbracket \rightarrow^{*} r \quad \Longleftrightarrow \quad \mathcal{C} \llbracket e_{2} \rrbracket \rightarrow^{*} r
$$

Two expressions are said to be semantically equivalent, if under all (closing) contexts, if one evaluates to an exception $r$, the other also evaluates to $r$.

\subsection{Crashing}

We use BAD to signal that something has gone wrong in the program: it has crashed.

DEFINITION 2 (Crash). A closed term e crashes iff $e \rightarrow^{*}$ BAD.
Our technique can only guarantee partial correctness: a diverging program does not crash.

DEFINITION 3 (Diverges). A closed expression e diverges, written $e \uparrow$, iff either $e \rightarrow^{*}$ UNR, or there is no value val such that $e \rightarrow^{*}$ val.

At compile-time, one decidable way to check the safety of a program is to see whether the program is syntactically safe.

DEFInITION 4 (Syntactic safety). A (possibly-open) expression e is syntactically safe iff $\mathrm{BAD} \notin_{s}$ e. Similarly, a context $\mathcal{C}$ is syntactically safe iff $\mathrm{BAD} \notin_{s} \mathcal{C}$.

The notation $\mathrm{BAD} \notin_{s} e$ means $\mathrm{BAD}$ does not syntactically appear anywhere in $e$, similarly for $\mathrm{BAD} \notin_{s} \mathcal{C}$. For example, $\lambda x . x$ is syntactically safe while $\lambda x$. (BAD, $x)$ is not.

DEFINITION 5 (Crash-free Expression). A (possibly-open) expression e is crash-free iff:

$$
\forall \mathcal{C} . \mathrm{BAD} \notin_{s} \mathcal{C} \text { and } \vdash \mathcal{C} \llbracket e \rrbracket::() \Rightarrow \mathcal{C} \llbracket e \rrbracket \not^{*} \mathrm{BAD}
$$

The notation $\vdash \mathcal{C} \llbracket e \rrbracket::()$ means $\mathcal{C} \llbracket e \rrbracket$ is closed and well-typed. By "closed" we mean that no variable is free in $\mathcal{C} \llbracket e \rrbracket$, not even a toplevel function like head. The quantified context $\mathcal{C}$ serves the usual role of a "probe" that tries to provoke $e$ into crashing. Notice that a crash-free expression may not be syntactically safe, for example:

$\backslash \mathrm{x}$. case $\mathrm{x} * \mathrm{x}>=0$ of $\{$ True $\rightarrow \mathrm{x}+1$; False $\rightarrow$ BAD $\}$

The tautology $x * x>=0$ is always true, so the BAD can never be reached. On the other hand, $(B A D, 3)$ is not crash-free because there exists a context, fst $\llbracket \bullet \rrbracket$, such that:

$$
\text { fst }(\mathrm{BAD}, 3) \rightarrow \mathrm{BAD}
$$

In short, crash-freeness is a semantic concept, and hence undecidable, while syntactic safety is syntactic and readily decidable. Certainly, a syntactically safe expression is crash-free:

Lemma 1 (Syntactically Safe Expression is Crash-free).

\begin{tabular}{|c|c|c|c|}
\hline $\begin{array}{l}t \\
t\end{array}$ & $\begin{array}{c}\in \\
::= \\
\mid\end{array}$ & $\begin{array}{l}\text { Contract } \\
\{x \mid p\} \\
x: t_{1} \rightarrow t_{2} \\
\left(t_{1}, t_{2}\right) \\
\text { Any }\end{array}$ & $\begin{array}{l}\text { Predicate Contract } \\
\text { Dependent Function Contract } \\
\text { Data Constructor Contract } \\
\text { Polymorphic Any Contract }\end{array}$ \\
\hline
\end{tabular}

$$
\text { e is syntactically safe } \Rightarrow \quad \text { e is crash-free }
$$

\section{Contract Syntax and Semantics}

Figure 3: Syntax of contracts

Having discussed the language of programs, we now discuss the language of contracts. Figure 3 gives their syntax. For reasons of notational simplicity, we restrict data constructor contracts to pairs only, but the idea generalises readily.

\subsection{Contract Satisfaction}

We give the semantics of contracts by defining " $e$ satisfies $t$ ", written $e \in t$, in Figure 4. This is a purely declarative specification of contract satisfaction, that says which terms satisfy a contract, without saying how a satisfaction check might be performed. We regard the ability to give a simple, declarative, programmer-accessible specification of contract satisfaction as very important, but it is a property that few related works share, with the notable and inspiring exception of [3]. As that paper says 


\begin{tabular}{|c|c|c|c|}
\hline \multicolumn{4}{|c|}{ For a well-typed expression $e$, define $e \in t$ thus: } \\
\hline$e \in\{x \mid p\}$ & $\Longleftrightarrow$ & $\begin{array}{l}e \uparrow \text { or }(e \text { is crash-free and } \\
\left.p[e / x] \nrightarrow^{*}\{\mathrm{BAD}, \mathrm{False}\}\right)\end{array}$ & [A1] \\
\hline$e \in x: t_{1} \rightarrow t_{2}$ & $\Longleftrightarrow$ & $\begin{array}{l}e \uparrow \text { or }\left(e \rightarrow^{*} \lambda x . e_{2} \text { and }\right. \\
\left.\forall e_{1} \in t_{1} .\left(e e_{1}\right) \in t_{2}\left[e_{1} / x\right]\right)\end{array}$ & [A2] \\
\hline$e \in\left(t_{1}, t_{2}\right)$ & $\Longleftrightarrow$ & $\begin{array}{l}e \uparrow \text { or }\left(e \rightarrow^{*}\left(e_{1}, e_{2}\right) \text { and }\right. \\
\left.e_{1} \in t_{1}, e_{2} \in t_{2}\right)\end{array}$ & [A3] \\
\hline$e \in$ Any & $\Longleftrightarrow$ & True & [A4] \\
\hline
\end{tabular}

Figure 4: Contract Satisfaction

The structure of a non-compositional semantics like [the Findler-Felleisen wrapping algorithm] is difficult to understand. With just Definition 1 [which says that a term satisfies a contract if its wrapping cannot crash] to hand, an answer to the question "Does $e$ satisfy $t$ ?" is not easy because it involves consideration of every possible context. Nor can we ignore this problem, since in our experience most people's intuition differs from [Definition 1].

To a first approximation, the rules in Figure 4 should be selfexplanatory. For example, $e$ satisfies $\{x \mid p\}$ if $p[e / x]$ evaluates to True. More interestingly, $e$ satisfies the (non-dependent) function contract $t_{1} \rightarrow t_{2}$ iff $\left(e e_{1}\right)$ satisfies $t_{2}$ for any term $e_{1}$ satisfying $t_{1}$. To get dependent function contracts we must simply remember to substitute $\left[e_{1} / x\right]$ in $t_{2}$. However, these definitions are carefully crafted at the edges, and we now discuss the less-obvious choices.

In Figure 4, both $e$ and $t$ may mention functions bound in the toplevel definitions $\Delta$. These functions are necessary for the evaluation relation of rule [A1] to make sense. To reduce clutter, we do not make these top-level bindings explicit, by writing $\Delta \vdash e \in t$, but instead allow rule [E-top] of Figure 2 to consult $\Delta$ implicitly.

\subsection{Only Crash-free Terms Satisfy Predicate Contracts}

The alert reader will notice that [A1] specifies that only crashfree terms satisfy a predicate contract $\{x \mid p\}$. This means that the contract $\{x \mid$ True $\}$, which we abbreviate to $0 \mathrm{k}$, is satisfied precisely by the crash-free terms. Even the identity function only guarantees a crash-free result if it is given a crash-free argument! Other choices are possible, but we postpone the discussion to $\S 5.4$, when we have more scaffolding in place.

\subsection{Diverging Terms}

The definitions in Figure 4 specify that a divergent term $e$ satisfies every contract. We made this choice because otherwise we would often have to prove termination in order to prove that $e \in t$. For example:

$$
f x=\text { if } x<10 \text { then } x \text { else } f(x / 2)
$$

Does $\mathrm{f} \in \mathrm{Ok} \rightarrow\{x \mid x<10\}$ ? The then branch clearly satisfies the postcondition but what about the else branch? Specifying that divergence satisfies any contract allows us to answer "yes" without proving termination. Furthermore, despite divergence, a caller of $f$ can still rely on $f$ 's postcondition:

$$
g y=\text { if }(f y>10) \text { then error "Urk" else True }
$$

Here g cannot crash, because $f$ guarantees a result less than 10 , or else diverges.

Our choice has the nice consequence that every contract is inhabited (by divergence). This matters. Consider whether ( $\lambda x . \mathrm{BAD})$ sat- isfies $\{x \mid$ False $\} \rightarrow$ ok. If $\{x \mid$ False $\}$ was uninhabited, the answer would be "yes", since [A2] holds vacuously. But that choice is incompatible with building a rigorous connection (sketched in $\$ 2.5$ ) between contract satisfaction and Findler-Felleisen-style wrapping. Indeed, Findler and Blume are forced to invent an awkward (and entirely informal) predicate form "non-empty-predicate" [10], which we do not need.

\subsection{The Any Contract}

If we only have [A1]-[A3], the expression BAD would not satisfy any contract, but we saw in $\S 2.2$ that this choice is too conservative for a lazy language. We therefore introduce a special contract, named Any, which is satisfied by any expression, including BAD (case [A4] in Figure 4). Now we can give a contract to fst:

$$
\begin{aligned}
& \{-\# \text { CONTRACT fst : : (Ok, Any) } \rightarrow 0 \mathrm{k} \#-\} \\
& \text { fst }(\mathrm{x}, \mathrm{y})=\mathrm{x}
\end{aligned}
$$

Any is also useful in post-conditions: a function whose postcondition is Any is a function that may crash. Haskell programmers often write packaged versions of Haskell's error function, such as

$$
\begin{aligned}
& \text { myError : : String } \rightarrow \text { a } \\
& \text { \{-\# CONTRACT myError : : Ok } \rightarrow \text { Any \#- } \\
& \text { myError } \mathrm{s}=\text { error ("Fatal error: " ++ s) }
\end{aligned}
$$

So BAD satisfies Any. In fact, BAD satisfies only the contract Any because it fails the constraints stated in [A1]-[A3]:

$$
\begin{array}{lll}
\text { BAD } & \notin & \text { (Any, Any }) \\
\text { BAD } & \notin & \text { Any } \rightarrow \text { Any }
\end{array}
$$

\subsection{Open Expressions}

We have mentioned that $e$ and $t$ may mention functions bound in the top-level environment. These functions participate in the evaluation of rule [A1]. But suppose that the programmer declares

$$
\begin{aligned}
& \{-\# \text { CONTRACT } f::\{x \mid x>0\} \rightarrow 0 k \#-\} \\
& f=\ldots
\end{aligned}
$$

When checking the contracts of a function $g$ that calls $f$, we should presumably assume only f's declared contract, without looking at its actual definition. Doing so is more modular, and allows the programmer to leave room for future changes by specifying a contract that is more restrictive than the current implementation.

This goal is easily achieved. Suppose the declared contracts for $f$ and $g$ are $t_{f}, t_{g}$ respectively, and the definition of $g$ is $g=e_{g}$ where $f$ is called in $e_{g}$. Then, instead of checking that $e_{g} \in t_{g}$, we check that

$$
\left(\lambda f . e_{g}\right) \in t_{f} \rightarrow t_{g}
$$

That is, simply lambda-abstract over any variables free in $e_{g}$ that have declared contracts. As an alternative, we also allow the programmer to omit a contract specification (just as type signatures are often omitted), in which case the contract checker selectively inlines the function when proving the correctness of its callers (for recursive functions see [39]). The exact details are a software engineering matter; our point here is that the underlying infrastructure allows a variety of choices.

The same technique simplifies the problem of checking satisfaction for recursive functions. If the programmer specifies the contract $t_{f}$ for a definition $\mathrm{f}=e$, then it suffices to check that

$$
\lambda f . e \in t_{f} \rightarrow t_{f}
$$

which is easier because $\lambda f . e$ does not call $f$ recursively. There is nothing new here - it is just the standard technique of loop invariants in another guise - but it is packaged very conveniently. 


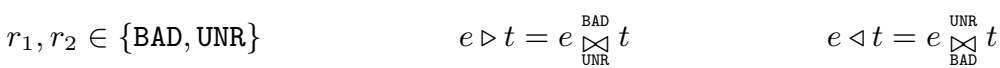

$$
\begin{aligned}
& e \underset{r_{2}}{\stackrel{r_{1}}{\bowtie}}\{x \mid p\}=e \text { 'seq' case }\left(\mathrm{fin}_{0} p[e / x]\right) \text { of }\left\{\text { True } \rightarrow e ; \text { False } \rightarrow r_{1}\right\} \\
& e \underset{r_{2}}{r_{1}} x: t_{1} \rightarrow t_{2}=e \text { 'seq' } \lambda v \cdot\left(\left(e\left(v \underset{r_{1}}{\stackrel{r_{2}}{\bowtie}} t_{1}\right)\right) \underset{r_{2}}{\stackrel{r_{1}}{\bowtie}} t_{2}\left[v \underset{r_{1}}{\stackrel{r_{2}}{\bowtie}} t_{1} / x\right]\right) \\
& e \underset{r_{2}}{\stackrel{r_{1}}{\bowtie}}\left(t_{1}, t_{2}\right)=\text { case } e \text { of }\left(x_{1}, x_{2}\right) \rightarrow\left(x_{1} \underset{r_{2}}{\stackrel{r_{1}}{\bowtie}} t_{1}, x_{2} \underset{r_{2}}{\stackrel{r_{1}}{\bowtie}} t_{2}\right) \\
& e_{r_{2}}^{r_{1}} \text { Any }=r_{2}
\end{aligned}
$$

Figure 5: Projection Definition

\section{Contract Checking}

So far we have a nice declarative specification of when a term $e$ satisfies a contract $t$. Of course, $e \in t$ is undecidable in general, but if we could statically check many (albeit not all) such claims, we would have a powerful tool. For example, if we could show that main $\in 0 \mathrm{k}$, then we would have proved that the entire program is crash-free.

In their ground-breaking paper [11], Findler \& Felleisen describe how to "wrap" a term in a contract-checking wrapper, that checks at run-time (a) that the term obeys its contract, and (b) that the context of the term respects the contract. How can we do the same at compile time? A promising approach, first suggested in [38] and sketched in $\S 2.5$, is to wrap the term in the Findler-Felleisen way, and check that the resulting term is crash free.

But is that sound? That is, does that prove that $e \in t$ ? What about the other way round? These questions are answered by our main theorem:

THEOREM 1 (Soundness and Completeness of Contract Checking). For all closed expressions $e$, and closed crash-free contracts $t$,

$$
(e \triangleright t) \text { is crash-free } \Longleftrightarrow \quad e \in t
$$

The form $(e \triangleright t)$ wraps $e$ in a Findler-Felleisen-style contract checker, specified in Figure 5. As in the case of contract satisfaction, there are tricky details, as we discuss in $\$ 5.1$. Another subtle but important point is the requirement that the contract $t$ be "crashfree"; this deals with contracts that crash and is discussed in $\S 6.1$.

The statement of Theorem 1 differs only in its technical details from soundness and completeness theorems in [3], although our proof technique is different to theirs. The reader may find a complete proof in [39].

The result is very strong. It states that the wrapped term $e \triangleright t$ is crash-free if and only if $e \in t$. Certainly, then, if we can prove that $e \triangleright t$ is crash-free, we have proved that $e \in t$. But how can we prove that $e \triangleright t$ is crash-free? That, in turn, is undecidable, but there are many useful approximations. For example, the approach we take is to perform meaning-preserving transformations on $e \triangleright t$, of precisely the kind that an optimising compiler might perform (inlining, $\beta$-reduction, constant folding, etc). If we can "optimise' (i.e. symbolically simplify) the term to a form that is syntactically safe ( $\$ 3.3)$, then we are done. The better the optimiser, the more contract satisfaction checks will succeed - but none of that affects Theorem 1. For details see [38].

\subsection{Wrapping}

Our goal is to define $e \triangleright t$ such that Theorem 1 holds. Figure 5 gives the definition of a single, general combinator $\bowtie$, and two notational abbreviations $\triangleright$ (pronounced "ensures") and $\triangleleft$ (pronounced "requires"). None of these operators are part of the syntax of ex- pressions (Figure 1); rather they are thought of as macros, which expand to a particular expression. Informally:

$$
e \underset{r_{2}}{\stackrel{r_{1}}{\bowtie} t} t
$$

is a term that behaves just like $e$, except that it throws exception $r_{1}$ if $e$ does not respect $t$, and throws exception $r_{2}$ if the wrapped term is used in a way that does not respect $t$. In the vocabulary of "blame", $r_{1}$ means "blame $e$ " while $r_{2}$ means "blame the context". Figure 5 defines the convenient abbreviations

$$
e \triangleright t=e \underset{\mathrm{UNR}}{\underset{\mathrm{BAD}}{\bowtie}} t \quad e \triangleleft t=e \underset{\mathrm{BAD}}{\underset{\mathrm{UNR}}{凶}} t
$$

So $e \triangleright t$ crashes (with BAD) if $e$ does not satisfy $t$, and diverges (with UNR) if the context does not respect $t$.

Temporarily ignoring the occurrences of "seq" and 'fin', the main structure of Figure 5 is standard from earlier works [11], and we do not belabour it here. In particular, note the inversion of $r_{1}$ and $r_{2}$ in the expansion of function contracts. The wrapping of Any, while new, is also obvious after a moment's thought. For example,

$$
\begin{aligned}
& \text { fst } \triangleright(\mathrm{Ok}, \text { Any }) \rightarrow \mathrm{Ok} \\
& =\lambda v \cdot((\mathrm{fst}(v \triangleleft(\mathrm{Ok}, \text { Any }))) \triangleright \mathrm{Ok}) \\
& =\lambda v \cdot((\text { fst }(v \triangleleft(\mathrm{Ok}, \text { Any })))) \\
& =\lambda v \cdot((\text { fst }(\text { case } v \text { of }(a, b) \rightarrow(a \triangleleft \mathrm{Ok}, b \triangleleft \text { Any })))) \\
& =\lambda v \cdot((\text { fst }(\text { case } v \text { of }(a, b) \rightarrow(a, \mathrm{BAD}))))
\end{aligned}
$$

Here we have used the fact that $e \bowtie 0 \mathrm{k}=e \bowtie\{x \mid$ True $\}=e$. That is, considered as a wrapper $0 \mathrm{k}$ does nothing at all. In this example we see that the wrapper replaces the second component of the argument to $f s t$ with BAD, so that if $f$ st should ever look at it, the program will crash. That is exactly right, because the contract says that the second component can be anything, with contract Any.

\subsection{The Use of seq}

The reader may wonder about the uses of seq in [P1] and [P2] of Figure 5. The function seq (short for "sequence") is defined as follows:

$$
e_{1} \text { 'seq' } e_{2}=\text { case } e_{1} \text { of }\left\{\text { DEFAULT } \rightarrow e_{2}\right\}
$$

It is necessary in the definition of $\bowtie$ to ensure that Theorem 1 holds for (a) divergent and (b) crashing terms. For example, if bot is a diverging term (defined by bot $=$ bot), then Figure 4 says that bot $\in\{x \mid$ False $\}$. But if [P1] lacked the seq, we would have

$$
\begin{aligned}
& \text { bot } \triangleright\{x \mid \text { False }\} \\
& =\text { case False of }\{\text { True } \rightarrow \text { bot; False } \rightarrow \text { BAD }\} \\
& =\text { BAD, which is not crash-free }
\end{aligned}
$$

thus contradicting Theorem 1. Dually, we must ensure that BAD $\notin$ $\mathrm{Ok} \rightarrow$ Any. Without the seq in [P2] we would get

$$
\begin{aligned}
\mathrm{BAD} \triangleright \mathrm{Ok} \rightarrow \mathrm{Any} & =\lambda v .((\mathrm{BAD}(v \triangleleft \mathrm{Ok})) \triangleright \text { Any }) \\
& =\lambda v . \text { UNR, } \quad \text { which is crash-free }
\end{aligned}
$$


again contradicting Theorem 1 . Have we covered all the cases? A quick check shows that for any contract $t, \mathrm{BAD} \notin t$ and UNR $\in t$, which is reassuring. More solidly, Theorem 1 goes through with the definitions of Figure 5.

\subsection{Properties of Contracts}

Our contract combinators possess many nice properties. We summarise these results in Figure 6. It took us some while to evolve a set of definitions for $\in, \bowtie$, etc that validated such crisp results. These lemmas form a basis for proving our main result: Theorem 1.

Figure 6 employs a useful ordering over expressions, called crashes more often:

DEFINITION 6 (Crashes more often). $e_{1}$ crashes more often than $e_{2}$, written $e_{1} \preceq e_{2}$, iff for all closing contexts $\mathcal{C}$

$$
\mathcal{C} \llbracket e_{2} \rrbracket \rightarrow^{*} \mathrm{BAD} \quad \Rightarrow \quad \mathcal{C} \llbracket e_{1} \rrbracket \rightarrow^{*} \mathrm{BAD}
$$

Informally $e_{1}$ crashes more often than $e_{2}$ if they behave in exactly the same way except that $e_{1}$ may crash when $e_{2}$ does not.

The proof of our main Theorem 1 is by induction on the size of the contract $t$. To give a flavour of the proof we show the only interesting case here, that for function contracts when $e \rightarrow^{*}$ val $\notin$ $\{\mathrm{BAD}, \mathrm{UNR}\}$. We give only the proof for non-dependent function contracts because the dependent case is more intricate and it is harder to see the wood for the trees. Full proofs can be found in [39]. The cf stands for "crash-free".

$$
\begin{aligned}
& e \triangleright t_{1} \rightarrow t_{2} \text { is } \mathbf{c f} \\
& \Longleftrightarrow \quad \text { (By definition of } \triangleright \text { ) } \\
& e^{\prime} \operatorname{seq}^{\prime} \lambda v \cdot\left(e\left(v \triangleleft t_{1}\right)\right) \triangleright t_{2} \text { is } \mathbf{c f} \\
& \Longleftrightarrow \quad\left(\text { Since } e \rightarrow^{*} \text { val } \notin\{\mathrm{BAD}, \mathrm{UNR}\}\right) \\
& \lambda v \cdot\left(e\left(v \triangleleft t_{1}\right)\right) \triangleright t_{2} \text { is } \mathbf{c f} \\
& \Longleftrightarrow \quad \text { (Property of crash-freeness) } \\
& \text { (†) } \forall \text { closed, cf } e^{\prime} .\left(e\left(e^{\prime} \triangleleft t_{1}\right)\right) \triangleright t_{2} \text { is cf }
\end{aligned}
$$

Now the proof splits into two. In the reverse direction, we start with the assumption $e \in t_{1} \rightarrow t_{2}$ :

$$
\begin{array}{ll} 
& e \in t_{1} \rightarrow t_{2} \\
\Rightarrow \quad & (\text { By defn of } e \in t) \\
& \forall e_{1} \in t_{1} \cdot\left(e e_{1}\right) \in t_{2} \\
\Rightarrow \quad & \text { (By Key Lemma (Figure 6)) } \\
& \forall \text { closed, cf } e^{\prime} .\left(e\left(e^{\prime} \triangleleft t_{1}\right) \in t_{2}\right. \\
\Longleftrightarrow \quad & \text { (By induction) } \\
(\dagger) \quad \forall \text { closed, cf } e^{\prime} .\left(e\left(e^{\prime} \triangleleft t_{1}\right)\right) \triangleright t_{2} \text { is cf }
\end{array}
$$

and now we have reached the desired conclusion $(\dagger)$. In the forward direction, we start with $(\dagger)$ :

$$
\begin{array}{ll}
(\dagger) \quad \forall \text { closed, cf } e^{\prime} .\left(e\left(e^{\prime} \triangleleft t_{1}\right)\right) \triangleright t_{2} \text { is cf } \\
\Rightarrow \quad & \text { (By induction, } \left.e_{1} \in t_{1} \Rightarrow e_{1} \triangleright t_{1} \text { is cf }\right) \\
& \forall \text { closed } e_{1} \in t_{1} .\left(e\left(\left(e_{1} \triangleright t_{1}\right) \triangleleft t_{1}\right)\right) \triangleright t_{2} \text { is cf } \\
\Rightarrow \quad & (\text { By Projection Pair and Congruence (Figure 6)) } \\
& \forall e_{1} \in t_{1} .\left(e e_{1}\right) \triangleright t_{2} \text { is cf } \\
\Rightarrow \quad & (\text { By induction }) \\
& \forall e_{1} \in t_{1} \cdot\left(e e_{1}\right) \in t_{2} \\
\Longleftrightarrow \quad & (\text { by definition of } \in) \\
& e \in t_{1} \rightarrow t_{2}
\end{array}
$$

There are two key steps in this short sequence. First, we choose a particular crash-free $e^{\prime}$, namely $\left(e_{1} \triangleright t_{1}\right)$ where $e_{1} \in t_{1}$. The second step is the appeal to the Projection Pair lemma, which itself is non-trivial.

\subsection{Why Only Crash-free Terms Satisfy Predicate Contracts}

In $\S 4.2$ we promised to explain why we chose to allow only crashfree terms to satisfy a predicate contract, regardless of the predicate. An obvious alternative design choice for contract satisfaction would be to drop the " $e$ is crash-free" condition in the predicate contract case:

$$
e \in\{x \mid p\} \Longleftrightarrow e \uparrow \text { or } p[e / x] \not^{*}\{\mathrm{BAD}, \text { False }\}
$$

Then we could get rid of Any, because $\{x \mid$ True $\}$ would do instead. On the other hand, a polymorphic contract meaning "crashfree" is extremely useful in practice, so we would probably need a new contract $\mathrm{Ok}$ (now not an abbreviation) defined thus:

$$
e \in \mathrm{Ok} \Longleftrightarrow e \text { is crash-free }
$$

This all seems quite plausible, but it has a fatal flaw: we could not find a definition for $\triangleright$ that validates our main theorem. That is, our chosen definition for $\in$ makes Figure 5 work out, whereas the otherwise-plausible alternative appears to prevent it doing so.

Suppose we have [B1] instead of [A1], that means (BAD, BAD) $\in$ $\{x \mid$ True $\}$. However, according to [P1], we have (BAD, BAD) $\triangleright$ $\{x \mid$ True $\}=(\mathrm{BAD}, \mathrm{BAD})$ which is not crash-free. This means Theorem 1 fails. Can we change [P1] to fix the theorem? It is hard to see how to do so. The revised rule must presumably look something like

$e \triangleright\{x \mid p\}=$ case $\mathrm{fin} p[e / x]$ of $\{$ True $\rightarrow ? ? ? ;$ False $\rightarrow$ BAD $\}$

But what can we put for "???"? Since $e \triangleright t$ is supposed to behave like $e$ if $p[e / x]$ holds, the "???" must be $e$ - but then BAD $\triangleright\{x \mid$ True $\}$ would not be crash free. This difficulty motivates our choice that predicate contracts are satisfied only by crash-free terms.

\section{Contracts that Crash}

Our goal is to detect crashes in a program with the help of contracts; we do not expect contracts themselves to introduce crashes. One approach, taken by Blume \& McAllester [3], is to prohibit a contract from mentioning any function that might crash. But that is an onerous restriction, as we argued in $\S 2.4$.

It is attractive simply to allow arbitrary crashes in contracts; after all, Figure 4 specifies exactly which terms inhabit even crashing contracts. Alas, if we drop the (still-to-be-defined) condition "crash-free contract" from Theorem 1, the $(\Rightarrow)$ direction still holds, but the $(\Leftarrow)$ direction fails. Here is a counter-example involving a crashing contract. We know that:

$$
\lambda x . x \in\{x \mid \mathrm{BAD}\} \rightarrow \mathrm{Ok}
$$

because the only expression that satisfies $\{x \mid \mathrm{BAD}\}$ is an expression that diverges and a diverging expression satisfies Ok. But we have:

$$
\begin{aligned}
\lambda x . x \triangleright\{x \mid \mathrm{BAD}\} \rightarrow \mathrm{O} \mathrm{k}= & \lambda v \cdot(\lambda x \cdot x(v \triangleleft\{x \mid \mathrm{BAD}\})) \\
= & \lambda v \cdot(v \triangleleft\{x \mid \mathrm{BAD}\}) \\
= & \lambda v \cdot(v \text { 'seq' } \mathrm{BAD}) \\
& \text { which is not crash-free }
\end{aligned}
$$

\subsection{Crash-free Contracts}

So unrestricted crashes in contracts invalidates (the $\Leftarrow$ direction of) Theorem 1. But no one is asking for unrestricted crashes! For example, this contract doesn't make much sense:

$$
t_{\text {bad }}=x s: 0 \mathrm{k} \rightarrow\{r \mid r>\text { head } x s\}
$$

What does $t_{b a d}$ mean if the argument list is empty? Much more plausible is a contract like this (see $\S 2.4)$ :

$$
t_{\text {good }}=x s:\{x s \mid \operatorname{not}(\operatorname{null} x s)\} \rightarrow\{r \mid r>\text { head } x s\}
$$




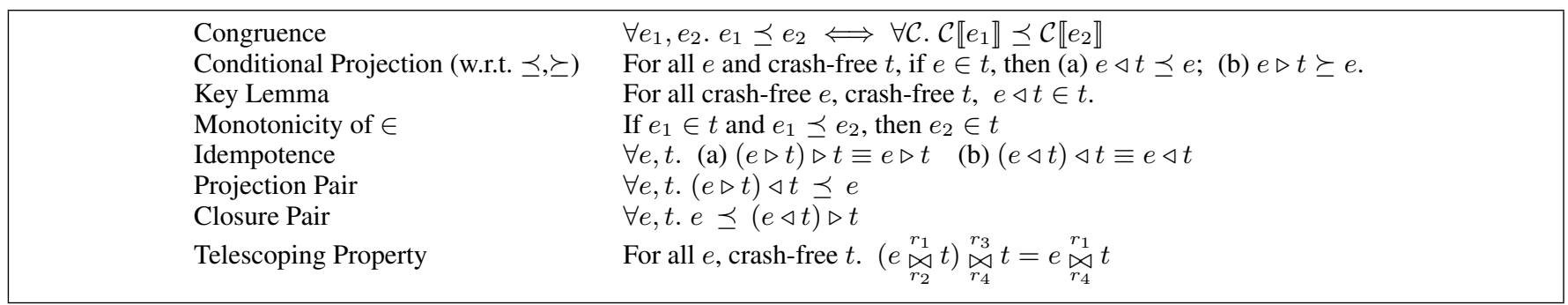

Figure 6: Properties of $\triangleright$ and $\triangleleft$

which specifies that the argument list is non-empty, and guarantees to return a result bigger than head of the argument. Thus motivated, we define a notation of a "crash-free" contract:

DEFINITION 7 (Crash-free Contract). A contract t is crash-free iff

$\quad t$ is $\{x \mid p\}$ and $p$ is crash-free
or $\quad t$ is $x: t_{1} \rightarrow t_{2}$ and $t_{1}$ is crash-free and
$\quad$ for all $e_{1} \in t_{1}, t_{2}\left[e_{1} / x\right]$ is crash-free
or $\quad t$ is $\left(t_{1}, t_{2}\right)$ and both $t_{1}$ and $t_{2}$ are crash-free
or $\quad t$ is Any

The definition is essentially the same as that of $T_{\text {safe }}$ in [3], although perhaps a little more straightforward. It simply asks that the predicates in a contract are crash-free under the assumption that the dependent function arguments satisfy their contracts. So, under this definition, $t_{b a d}$ is ill-formed while $t_{\text {good }}$ is crash-free. The latter is crash-free because head $x s$ is crash-free for every $x s$ that satisfies not (null $x s$ ).

\subsection{Wrapping dependent function contracts}

Recall [P2] from Figure 5:

$e \underset{r_{2}}{r_{1}} x: t_{1} \rightarrow t_{2}=e^{\prime} \mathrm{seq}^{\prime} \lambda v \cdot\left(\left(e\left(v \underset{r_{1}}{\stackrel{r_{2}}{\bowtie}} t_{1}\right)\right) \underset{r_{2}}{\underset{r_{1}}{\bowtie}} t_{2}\left[\left(v \underset{r_{1}}{\underset{r_{2}}{\bowtie}} t_{1}\right) / x\right]\right)$

Notice that $v$ is wrapped by $v \underset{r_{1}}{r_{2}} t_{1}$ even in the contract $t_{2}$, as well as in the argument to $e$. Could we simplify [P2] by omitting this wrapping, thus?

$$
e \underset{r_{2}}{\stackrel{r_{1}}{\bowtie}} x: t_{1} \rightarrow t_{2}=e^{\prime} \mathrm{seq}^{\prime} \lambda v \cdot\left(\left(e\left(v \underset{r_{1}}{\stackrel{r_{2}}{\bowtie}} t_{1}\right)\right) \underset{r_{2}}{\stackrel{r_{1}}{\bowtie}} t_{2}[v / x]\right)
$$

No, we could not: Theorem 1 would fail again. Here is a counterexample.

$$
\begin{aligned}
& \{-\# \text { CONTRACT } \mathrm{h}::\{\mathrm{x} \mid \operatorname{not}(\text { null } \mathrm{x})\} \\
& \rightarrow\{\mathrm{z} \mid \text { head } \mathrm{x}==\mathrm{z}\} \#-\}
\end{aligned}
$$

Now $\mathrm{h}$ satisfies its contract $t_{h}$, but $\mathrm{h} \triangleright t_{h}$ is not crash-free, as the reader may verify.

We remarked earlier that Blume \& McAllester require that contracts only call crash-free functions. But the wrapping of $v$ inside $t_{2}$ in rule [P2] might itself introduce crashes, at least if $t_{2}$ uses $x$ in a way that does not respect $t_{1}$. They therefore use another variant of [P2], as follows:

$$
e \underset{r_{2}}{\stackrel{r_{1}}{凶}} x: t_{1} \rightarrow t_{2}=e^{\text {'seq' }} \lambda v \cdot\left(\left(e\left(v \underset{r_{1}}{\underset{r_{2}}{凶}} t_{1}\right)\right) \underset{r_{2}}{\underset{r_{1}}{\bowtie}} t_{2}\left[\left(v \underset{\mathrm{UNR}}{r_{2}} t_{1}\right) / x\right]\right)
$$

Notice the "UNR" introduced out of thin air in the wrapping of $v$ in $t_{2}$, which is enough to maintain their no-crashing invariant. Happily, if the contracts are crash-free (which we need anyway, so that it is possible to call head) there is no need for this somewhat ad-hoc fix.

\subsection{Practical consequences}

One might worry that the crash-freeness condition in Theorem 1 makes the verification task more onerous: perhaps to prove $e \in t$ now we must check two things (a) that $t$ is crash-free formed and (b) that $e \triangleright t$ is crash-free. Happily, this is not necessary, because the $(\Rightarrow)$ of Theorem 1 holds for arbitrary $t$ :

THEOREM 2. For all closed expression e, for all contract $t$,

$$
(e \triangleright t) \text { is crash-free } \Rightarrow e \in t
$$

The proof of Theorem 2 is the same as the proof for the direction $(\Rightarrow)$ of Theorem 1 because only the proof for the direction $(\Leftarrow)$ of Theorem 1 requires the condition that $t$ to be crash-free.

\section{Contracts that diverge}

Our system allows non-termination both in the programs we verify, and in their specifications (contracts), which is most unusual for a system supporting static verification.

As we discussed in $\S 4.3$, we allow non-termination for programs because we work with a real-life programming language, in which many functions actually do not terminate. We do not want to exclude non-termination in general, even for specifications, because we do not want to be forced to perform termination proofs. Since the current advances in automatic termination proofs are still limited, especially for lazy programs, requiring termination would put a substantial extra burden on the user of our system.

What about divergent contracts? Many program verification systems for functional programming, such as HOL, systems based on dependent types (Coq, Agda), and ACL2, do not allow any nonterminating definitions. The main reason is that divergent terms introduce an immediate unsoundness in these systems. For example, by an (unsound) induction proof, a constant defined as let $\mathrm{x}=\mathrm{x}$ could be proven equal to both 1 and 2 , concluding that $1=2$.

But it would be onerous to insist that all contracts terminate, because the programmer can write arbitrary Haskell in contracts, and proving termination of arbitrary Haskell programs is hard. Furthermore, allowing non-termination in specifications is of direct benefit. Consider a function zipE which requires two inputs to have the same length:

$$
\begin{aligned}
& \text { \{-\# CONTRACT zipE :: xs:Ok } \\
& \text { zipE } \rightarrow \text { ys | sameLen xs ys\} } \rightarrow 0 k \#-\} \\
& \operatorname{zipE}[] \quad[] \quad=[] \\
& \operatorname{zipE}(x: x s)(y: y s)=(x, y): z i p E x s \text { ys } \\
& \text { zipE _ _ n e error 'unequal lengths', } \\
& \text { sameLen [] [] = True } \\
& \text { sameLen }(\mathrm{x}: \mathrm{xs})(\mathrm{y}: \mathrm{ys})=\text { sameLen } \mathrm{xs} \text { ys } \\
& \text { sameLen _ _ _ = False }
\end{aligned}
$$

Here, two infinite lists satisfy the contract for ys, since (sameLen xs ys) diverges, and indeed zipE does not crash for such argu- 
ments. Care is necessary in writing the contract: if we had instead said length $\mathrm{xs}==$ length ys, the contract would diverge if only one argument was infinite, but zipE would crash for such arguments, so it would not satisfy this alternative contract. In this way, safety properties over infinite structures are allowed. (Safety properties are properties that always have finite counter-examples whenever there exists any counter-example.)

Why is our approach sound? First, any contract we verify for a program only deals with partial correctness. In other words, all contracts are inhabited by non-terminating programs as well. Second, our system does not include reasoning mechanisms like equational reasoning. In fact, our system has no means of expressing equality at all! Everything is expressed in terms of Haskell expressions evaluating to boolean values, crashing, or not-terminating. Third, any specification that does not terminate is semantically the same as a True contract. Why? Because of the mysterious fin construct, as we discuss next.

\subsection{Using $f$ in in contract wrappers}

Suppose we have the top-level definition bot = bot; that is, bot diverges. Now consider $e=(\mathrm{BAD}, \mathrm{BAD})$ and $t=\{\mathrm{x} \mid$ bot $\}$. Then $e \notin t$ (since $e$ is not crash-free). If we did not use $f$ in in the definition of $\bowtie$ (Figure 5), $e \triangleright t$ would reduce to this term:

case bot of $\{$ True $\rightarrow$ (BAD, BAD); False $\rightarrow$ BAD \}

This term is contextually equivalent to bot itself, and so it is crashfree, contradicting our main theorem $(\S 5)$.

What to do? Execution has gotten stuck evaluating the diverging contract, and has thereby missed crashes in the term itself. Our solution is to limit the work that can be spent on contract evaluation. The actual definition of $\bowtie$ makes $e \triangleright t$ equal to

$$
\begin{aligned}
& \text { case (fin } \text { fot }_{0} \text { of } \\
& \quad \text { \{ True } \rightarrow \text { (BAD, BAD); False } \rightarrow \text { BAD }\}
\end{aligned}
$$

The operational semantics of $f$ in (Figure 2) gives a finite $M$ units of "fuel" to each $f i n$. Each reduction under a $f$ in increases the subscript on the fin until it reaches the maximum $M$ (rule [Efin1]). When the $\mathrm{f}$ in subscript $n$ reaches the limit $M$, fin gives up and returns True (rule [E-fin4]). Now, in the above example, for any finite $M$, we have

$$
e \triangleright t \rightarrow^{*}(\mathrm{BAD}, \mathrm{BAD})
$$

So we define our full-scale reduction relation $\rightarrow{ }^{*}$ in terms of $\rightarrow{ }_{M}^{*}$ : DEFINITION 8. We say that $e \rightarrow^{*}$ val iff there exists $N$ such that for any $M \geq N$ we have $e \rightarrow_{M}^{*}$ val.

Under this definition, $e \triangleright t \rightarrow^{*}$ (BAD, BAD), and Theorem 1 holds.

\subsection{Practical consequences}

This may all seem a bit complicated or artificial, but it is very straightforward to implement. First, remember that we are concerned with static verification, not dynamic checking. Uses of $f$ in are introduced only to check contract satisfaction, and are never executed in the running program. Second, our technique to check that $e \triangleright t$ is crash-free is to optimise it and check for syntactic safety. To be faithful to the $\rightarrow$ * semantics, we need only refrain from "optimising" (case ( $f$ in bot) of $\langle a l t s\rangle$ ) to bot, thereby retaining any BADs lurking in $\langle a l t s\rangle$. Since this particular optimisation is a tricky one anyway, it is quite easy to omit! In other words, in our static contract checking, we can safely omit $f$ in and the rules [E-fin1-4].

By being careful with our definition of $\rightarrow^{*}$, we can retain Theorem 1 in its full, bi-directional form. This approach is, of course, only available to us because we are taking a static approach to verification. A dynamic checker cannot avoid divergence in contracts (since it must evaluate them), and hence must lose the $(\Rightarrow)$ direction of Theorem 1, as indeed is the case in [3]. To put it another way, should our static checker fail, we cannot have recourse to a dynamic check (as happens in hybrid systems), because if the contract can diverge an otherwise perfectly correct program might diverge because the dynamic check loops. In any dynamic system, the run-time checks must terminate if they are to avoid changing the program semantics.

\section{Error Reporting}

Given $f \in t$, we have shown that to check $f \in t$, we check " $f \triangleright t$ is crash-free" instead. What happens if $f \triangleright t$ is not crash-free? That means when we try to optimize the term $f \triangleright t$ to some $e^{\prime}$ and there are some residual BADs in $e^{\prime}$, what we can report to the programmer from the contract violation?

To know which function to blame [11], we need to give each BAD a tag. That is BAD $l b l$ where the label $l b l$ is a function name. For a function $f$ with contract $t$, we check $f \triangleright t$ is crash-free or not, where

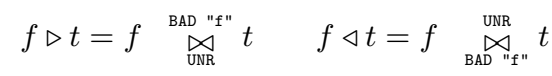

A residual BAD " $f$ " tells us that we should blame $f$. That means assuming $f$ takes arguments satisfying their corresponding preconditions, $f$ fails to produce a result that meets its postcondition. For example:

$$
\begin{aligned}
& \{-\# \text { CONTRACT inc }::\{x \mid x>0\} \rightarrow\{z \mid z>x\} \#-\} \\
& \text { inc } x=x-1
\end{aligned}
$$

after optimizing (i.e. simplifying) inc $\triangleright t_{\text {inc }}$, we have:

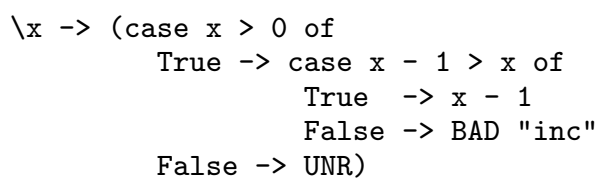

We can report to the programmer at compile-time:

$$
\begin{aligned}
& \text { Error: inc fails its postcondition } \\
& \text { when } x>0 \text { holds } \\
& x-1>x \text { does not hold }
\end{aligned}
$$

This error message is generated directly from the path that leads to the BAD "inc".

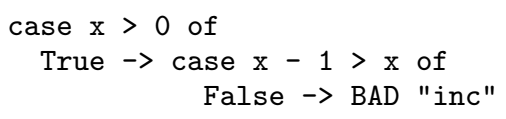

How about precondition violation? Suppose we know $f \in t_{f}$. If $f$ is called in a function $g$ with contract $t_{g}$, recalling the reasoning in $\S 4.5$, we shall check $\left(\lambda f . e_{g}\right) \in\left(t_{f} \rightarrow t_{g}\right)$. That means we check: $\left(\lambda f . e_{g}\right) \triangleright\left(t_{f} \rightarrow t_{g}\right)$ is crash-free or not. By the definition of $\triangleright$, we have: $\lambda f$. $\left(\left(e_{g}\left(f \triangleleft t_{f}\right)\right) \triangleright t_{g}\right)$.

In order to trace "which function calls which function that fails which function's precondition", instead of using $\left(f \triangleleft t_{f}\right)$, we use:

$$
\text { Inside lbl loc }\left(f \triangleleft t_{f}\right)
$$

The $l b l$ is the function name ( $f$ " in this case) and the loc indicates the location (e.g. (row,column)) of the definition of $f$ in the source file.

For example, we have:

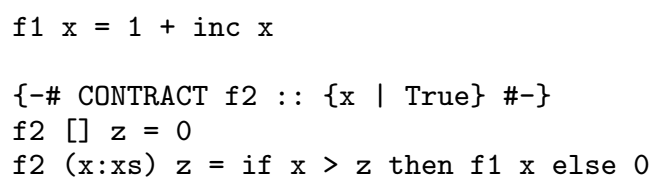


In our system, since $\mathrm{f} 1$ lacks a contract, we inline it at every call site, thus avoiding the necessity of supplying a contract for many trivial functions. (For recursive functions see [39].) After optimizing $f 2 \triangleright\{x \mid$ True $\}$, we have:

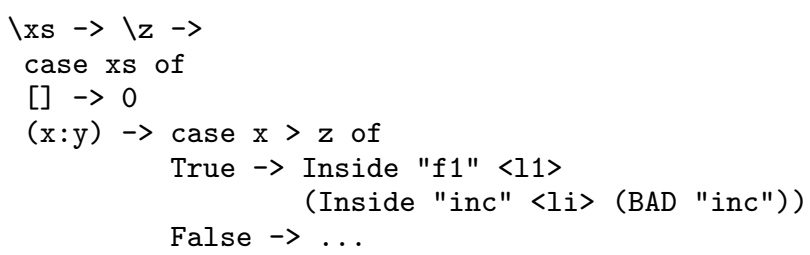

Note that, the "inc" in (BAD "inc") indicates which function's precondition is not fulfilled. Thus, the residual fragment enables us to give one counter-example with the following meaningful message at compile-time:

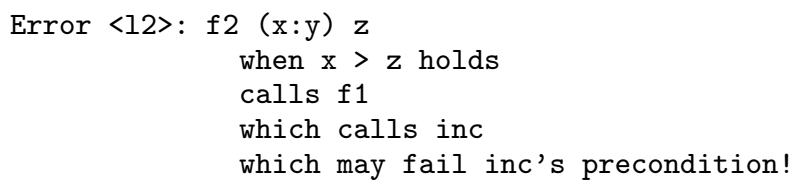

where the location $\langle 12\rangle$ indicates the location of the definition of $\mathrm{f} 2$ in the source file.

This error tracing technique is adapted from ESC/Haskell [38], which achieves the same goal as that in [23] but in a much simpler way. However, unlike [23] we have not yet formalised the correctness of its blame assignment.

\section{Related Work}

Static verification of software is a field dense with related work, of which we can only summarise a limited fraction here.

\subsection{Type systems}

In the functional language community, type systems have played significant roles in guaranteeing better software safety. Advanced type systems, such as dependent types, have been advocated to capture stronger properties. While full dependent type system (such as Cayenne [1]) is undecidable in general, $\mathrm{Xi}$ and Pfenning [37] have designed a smaller fragment based on indexed objects drawn from a constraint domain $\mathcal{C}$ whose decidability closely follows that of the constraint domain. Typical examples of objects in $\mathcal{C}$ include linear inequalities over integers, boolean constraints, or finite sets. In a more recent Omega project [32], Sheard shows how extensible kinds can be built to provide a more expressive dependent-style system. In comparison, our approach is much more expressive and programmer friendly as we allow arbitrary functions to be used in the pre/post annotations without the need to encode them as types. It is also easier for programmers to add properties incrementally. Moreover, our symbolic evaluation is formulated to adhere to lazy semantics and is guaranteed to terminate when code safety is detected or when a preset bound on the unrollings of each recursive function is reached. Compared with the dependent type approaches $[37,6,32,36]$ in general, we separate type and contract declarations so that type related work (e.g. type inference) and contract related techniques can be developed independently.

In Hoare Type Theory (HTT) [29, 28], higher-order predicates and recursive predicates can be used in specifications. Another work along this line is [31]. We allow higher-order functions and recursive functions to be used in contracts so in terms of these two aspects, we share the same expressiveness. As we use symbolic execution, inlining and induction make the job of verifying such contracts easier compared with theorem proving higher-order predicates and recursive predicates. But our contracts do not contain quantifiers while quantifiers are supported in [29, 28, 31].

\subsection{Extended static checking}

In an inspiring sequence of papers [22, 14, 13, 4], Leino, Nelson, Flanagan, and their colleagues showed the feasibility of applying an extended static checker to Modula-3 and then Java. Since then, several other similar systems have been further developed, including Spec\#'s and its automatic verifier Boogie [2] that is applicable to the C\# language. We adapt the same idea of allowing programmers to specify properties about each function (in the Haskell language) with pre/post annotations, but also allow pre/post annotations to be selectively omitted where desired. Furthermore, unlike previous approaches based on verification condition (VC) generation which rely solely on a theorem prover to verify, we use an approach based on symbolic evaluation that can better capture the intended semantics of a more advanced lazy functional language. With this, our reliance on the use of theorem provers is limited to smaller fragments that involve the arithmetical parts of expressions. Symbolic evaluation gives us much better control over the process of the verification where we have customised sound and effective simplification rules that are augmented with counter-example guided unrolling. More importantly, we are able to handle specifications involving recursive functions and/or higher-order functions which are not supported by either ESC/Java or Spec\#.

\subsection{Contracts}

The idea of "contract" was first established by Parnas [30] and popularized by Meyer in its use in Eiffel [24]. More recently, Findler and Felleisen introduced the notion of higher-order contracts, including a careful treatment of "blame" [11]. This paper unleashed a series of papers about contract checking in higher-order languages, including $[3,10,3,18,35,12,21,20,16]$. Although they share a common foundation, these papers differ in their notation and approach, which makes like-for-like comparisons difficult.

Of these papers, the work of Blume and McAllester [3] is by far the most closely related because they give a declarative semantics for contract satisfaction, and prove a connection with the dynamic wrappers of Findler and Felleisen. Here is a brief summary of the differences between some of this work, especially [3], and our own:

- We aim at static contract checking, for a statically typed language, whereas most of the related work deals with dynamic checks, or a hybrid checking strategy for a dynamically typed language.

- We deal with a lazy language; all other related work is for a strict languages. In particular, we give a crashing expression a contract Any while a contract is only given to non-crashing expressions in $[11,3,10]$.

- We deal with dependent function contracts which [10] does not.

- We lay great emphasis on crashing and diverging contracts, which are either not the focus of these other works, or are explicitly excluded. Our solution appears less restrictive than [3], by allowing crashing functions to be called within contracts ("non-total contracts" in their terminology), while still supporting Theorem 1 in both directions, rather than the less-useful $(\Leftarrow)$ direction only.

- The telescoping property (Figure 6) is first discovered in [3], but it does not seem to be used in any of their proofs, while we use it intensively to make many proofs much simpler.

- Findler and Blume discovered that contracts are pairs of projections in $[9,10]$. That means given a contract $t, \lambda e . \mathcal{W}_{t}(e)$ is a projection where $\mathcal{W}_{t}$ is a wrapper function. To be a projection w.r.t. $\sqsubseteq$, a function $p$ must satisfy these two properties:

$$
\text { 1. } p \circ p=p \text { (idempotence) }
$$


2. $p \sqsubseteq 1$ (result of projection contains no more information than its input)

Our $(\bullet \triangleright t)$ and $(\bullet \triangleleft t)$ (i.e. $\lambda x .(x \triangleright t)$ and $\lambda x .(x \triangleleft t))$ satisfy the idempotence property as shown in Figure 6, but does not satisfy (2). They only satisfy (2) under the condition that the input of the projection satisfies its contract $t$ as shown in Figure 6. Moreover, we discover the projection pair property (in Figure 6), which plays a crucial role in our proof.

- Blume \& McAllester dealt with recursive contracts, which we do not.

Inspired by [11, 3], Hinze et al. [18] implement contracts as a library in Haskell and contracts are checked at run-time. The framework also support contract constructors such as pairs, lists, etc. Another dynamic contract checking work is the Camila project [34] which use monads to encapsulate the pre/post-conditions checking behaviour.

The hybrid contract checking framework [12, 21, 20, 16], in theory, can be as powerful as our system. (Hybrid checking means a combination of static and dynamic contract checking.) But in practice, our symbolic execution strategy adopted from [38] gives more flexibility to the verification as illustrated in $\S 2.1$. In [35], Wadler and Findler show how contracts fit with hybrid types and gradual types by requiring casts in the source code. The casts are similar to the job of our $\triangleright$ and $\triangleleft$.

\subsection{Other work}

In [19], a compositional assertion checking framework has been proposed with a set of logical rules for handling higher-order functions. Given arguments satisfying their precondition, they check whether function definition satisfies its postcondition and the checking is currently a manual proof based on the logical rules. Apart from our focus on automatic verification, we can give precise blame when a contract violation is detected. The work in [19] has the strength in verification, but not in assigning blames.

Amongst the Haskell community, there have been several works that are aimed at providing high assurance software through validation (testing) [5], program verification (Programatica project [17]) or a combination of the two [8]. In the Programatica project, PLogic has been introduced to specify properties for Haskell programs. P-Logic allows programmers to express relations among multiple functions and it is more comprehensive then contracts, which only allow programmers to specify pre/postcondition of one function. The design of P-Logic focuses on proof construction while our focus is to find the right function to blame based on contract violation. Certainly, the more properties we can prove, the more precise the contract violation checking will be. Some design of P-Logic could be adopted in future.

Our approach eliminates the effort of inventing and learning a new logic together with its theorem prover. Furthermore, our verification approach does not conflict with the validation assisted approach used by $[5,8]$ and can play complementary roles.

Mitchells's Catch system statically infers the possibility of pattern matching failure, without any help from the programmer [26, 25]. The domain of a function is described by a language of regular expressions, which is incomparable with our contract language, and the technical details are very different. Moreover, Catch applies to first-order programs, so can be used only after a whole-program firstification transformation has removed (most of) the higher-order functions. In contrast, we analyse higher-order functions directly, in a modular (not whole-program) way.

\section{Conclusion and Future Work}

We have presented a sound and automatic static verification tool for a functional language, Haskell. Based on contracts and symbolic execution, our approach gives precise blame assignments at compile-time in the presence of higher-order functions and laziness. We lay particular emphasis on allowing the programmer to use all of Haskell in contracts, including functions that may crash or diverge.

We have developed a prototype implementation in the context of the Glasgow Haskell Compiler. It can prove some simple contract satisfaction checks, but is still incomplete for more sophisticated examples involving the use of recursive functions in predicates, such as sorting or AVL trees. Such predicates are also outside the reach of other static checkers, but they are important in Haskell programs. The shortcoming lies in the optimiser ( 55 ) and, in particular, the choice of precisely when to inline a function. We have manual proofs of many of our motivating examples (including sorting and AVL trees), and are working on heuristics to enable the optimiser to prove them too.

In future, we may introduce conjunctive/disjunctive contracts, recursive contracts, and contracts with quantifiers. Another important direction is to offer ways in which the programmer can help the system by suggesting suitable lemmas.

\section{Acknowledgments}

We would like to thank Philip Wadler, Robby Findler, Matthias Felleisen, and the anonymous referees for their careful comments. This work was partially supported by Microsoft Research through its $\mathrm{Ph}$.D. Scholarship program.

\section{References}

[1] Lennart Augustsson. Cayenne - language with dependent types. In Proceedings of the third ACM SIGPLAN international conference on Functional programming, pages 239250, New York, NY, USA, 1998. ACM.

[2] Mike Barnett, K. Rustan M. Leino, and Wolfram Schulte. The Spec\# programming system: An overview. CASSIS, LNCS 3362, 2004.

[3] Matthias Blume and David McAllester. Sound and complete models of contracts. J. Funct. Program., 16(4-5):375-414, 2006.

[4] L. Burdy, Y. Cheon, D. Cok, M. Ernst, J. Kiniry, G. Leavens, K. Leino, and E. Poll. An overview of JML tools and applications, 2003.

[5] Koen Claessen and John Hughes. Specification-based testing with QuickCheck. In Fun of Programming, Cornerstones of Computing, pages 17-40. Palgrave, March 2003.

[6] Sa Cui, Kevin Donnelly, and Hongwei Xi. ATS: A language that combines programming with theorem proving. In Bernhard Gramlich, editor, FroCos, volume 3717 of Lecture Notes in Computer Science, pages 310-320. Springer, 2005.

[7] Rowan Davies. Refinement-type checker for standard ML. In AMAST '97: Proceedings of the 6th International Conference on Algebraic Methodology and Software Technology, pages 565-566, London, UK, 1997. Springer-Verlag.

[8] Peter Dybjer, Qiao Haiyan, and Makoto Takeyama. Verifying Haskell programs by combining testing and proving. In Proceedings of Third International Conference on Quality Software, pages 272-279. IEEE Press, 2003. 
[9] R. B. Findler, M. Blume, and M. Felleisen. An investigation of contracts as projections. Technical report, University of Chicago Computer Science Department, 2006. Technical Report TR-2004-02.

[10] Robert Bruce Findler and Matthias Blume. Contracts as pairs of projections. In Functional and Logic Programming, pages 226-241. Springer Berlin / Heidelberg, 2006.

[11] Robert Bruce Findler and Matthias Felleisen. Contracts for higher-order functions. In Proceedings of the seventh ACM SIGPLAN international conference on Functional programming, pages 48-59, New York, NY, USA, 2002. ACM.

[12] Cormac Flanagan. Hybrid type checking. In Morrisett and Peyton Jones [27], pages 245-256.

[13] Cormac Flanagan, K. Rustan M. Leino, Mark Lillibridge, Greg Nelson, James B. Saxe, and Raymie Stata. Extended static checking for Java. In PLDI '02: Proceedings of the ACM SIGPLAN 2002 Conference on Programming language design and implementation, pages 234-245, New York, NY, USA, 2002. ACM.

[14] Cormac Flanagan and James B. Saxe. Avoiding exponential explosion: generating compact verification conditions. In POPL '01: Proceedings of the 28th ACM SIGPLAN-SIGACT Symposium on Principles of Programming Languages, pages 193-205, New York, NY, USA, 2001. ACM.

[15] Tim Freeman and Frank Pfenning. Refinement types for ML. In PLDI '91: Proceedings of the ACM SIGPLAN 1991 conference on Programming language design and implementation, pages 268-277, New York, NY, USA, 1991. ACM.

[16] Jessica Gronski and Cormac Flanagan. Unifying hybrid types and contracts. In Eighth Symposium on Trends in Functional Programming, April 2007.

[17] Thomas Hallgren. Haskell tools from the Programatica project. In Haskell '03: Proceedings of the 2003 ACM SIGPLAN workshop on Haskell, pages 103-106, New York, NY, USA, 2003. ACM.

[18] Ralf Hinze, Johan Jeuring, and Andres Löh. Typed contracts for functional programming. In Functional and Logic Programming: 8th International Symposium, pages 208-225, 2006.

[19] Kohei Honda and Nobuko Yoshida. A compositional logic for polymorphic higher-order functions. In PPDP '04: Proceedings of the 6th ACM SIGPLAN international conference on Principles and practice of declarative programming, pages 191-202, New York, NY, USA, 2004. ACM.

[20] Kenneth Knowles and Cormac Flanagan. Type reconstruction for general refinement types. In ESOP '07: Programming Languages and Systems, 16th European Symposium on Programming. Springer-Verlag, April 2007.

[21] Kenneth Knowles, Aaron Tomb, Jessica Gronski, Stephen N. Freund, and Cormac Flanagan. SAGE: Unified hybrid checking for first-class types, general refinement types, and dynamic (extended report). Technical report, UC Santa Cruz, 2006. http://sage.soe.ucsc.edu/sage-tr.pdf.

[22] K. Rustan M. Leino and Greg Nelson. An extended static checker for Modula-3. In CC '98: Proceedings of the 7th International Conference on Compiler Construction, pages 302-305, London, UK, 1998. Springer-Verlag.

[23] Philippe Meunier, Robert Bruce Findler, and Matthias Felleisen. Modular set-based analysis from contracts. In Morrisett and Peyton Jones [27], pages 218-231.
[24] Bertrand Meyer. Eiffel: The Language. Prentice Hall International, London, 1992.

[25] Neil Mitchell. Transformation and Analysis of Functional Programs. PhD thesis, University of York, 2008.

[26] Neil Mitchell and Colin Runciman. Not all patterns, but enough: an automatic verifier for partial but sufficient pattern matching. In Haskell '08: Proceedings of the first ACM SIGPLAN symposium on Haskell, pages 49-60, New York, NY, USA, 2008. ACM.

[27] J. Gregory Morrisett and Simon L. Peyton Jones, editors. POPL '06: Proceedings of the 33rd ACM SIGPLANSIGACT Symposium on Principles of Programming Languages, Charleston, South Carolina, USA, January 11-13, 2006. ACM, 2006.

[28] Aleksandar Nanevski, Amal Ahmed, Greg Morrisett, and Lars Birkedal. Abstract predicates and mutable ADTs in Hoare Type Theory. In Rocco De Nicola, editor, ESOP, volume 4421 of Lecture Notes in Computer Science, pages 189-204. Springer, 2007.

[29] Aleksandar Nanevski, Greg Morrisett, and Lars Birkedal. Polymorphism and separation in hoare type theory. In Proceedings of the 11th ACM SIGPLAN International Conference on Functional Programming, Portland, Oregon, USA, Sept., pages 62-73, 2006.

[30] D. L. Parnas. A technique for software module specification with examples. Communications of the ACM, 15(5):330-336, May 1972.

[31] Yann Régis-Gianas and François Pottier. A Hoare logic for call-by-value functional programs. In P Audebaud and C Paulin-Mohring, editors, MPC, volume 5133 of Lecture Notes in Computer Science, pages 305-335. Springer, 2008.

[32] Tim Sheard. Languages of the future. In OOPSLA '04: Companion to the 19th annual ACM SIGPLAN conference on Object-oriented programming systems, languages, and applications, pages 116-119, New York, NY, USA, 2004. ACM.

[33] The GHC Team. The Glasgow Haskell Compiler user's guide. www . haskell.org/ghc/documentation.html, 1998.

[34] J Visser, J. N. Oliveira, Barbosa L. S., J. F. Ferreira, and A. Mendes. CAMILA revival: VDM meets Haskell. In Nico Plat and Peter Gorm Larsen, editors, Overture Workshop (colocated with FM'05), 2005.

[35] Philip Wadler and Robert Bruce Findler. Well-typed programs can't be blamed. In Workshop on Scheme and Functional Programming, Sept 2007.

[36] Edwin M. Westbrook, Aaron Stump, and Ian Wehrman. A language-based approach to functionally correct imperative programming. In Proceedings of the 10th ACM SIGPLAN International Conference on Functional Programming, Tallinn, Estonia, pages 268-279, Sept. 2005.

[37] Hongwei Xi and Frank Pfenning. Dependent types in practical programming. In POPL '99: Proceedings of the 26th ACM SIGPLAN-SIGACT symposium on Principles of programming languages, pages 214-227, New York, NY, USA, 1999. ACM.

[38] Dana N. Xu. Extended static checking for Haskell. In Haskell '06: Proceedings of the 2006 ACM SIGPLAN workshop on Haskell, pages 48-59, New York, NY, USA, 2006. ACM.

[39] Na Xu. Static Contract Checking for Haskell. PhD thesis, University of Cambridge, 2008. http://www.cl.cam.ac . uk/techreports/. 